\title{
Molecular Building Blocks and Their Architecture in Biologically/Environmentally Compatible Soft Matter Chemical Machinery
}

\author{
Taro Toyota ${ }^{1,2,3 *}$, Taisuke Banno ${ }^{1}$, Sachiko $\mathrm{Nitta}^{4}$, Masahiro Takinoue ${ }^{3,5}$, \\ Tomonori Nomoto ${ }^{6}$, Yuno Natsume ${ }^{7}$, Shuichi Matsumura ${ }^{8}$ and Masanori Fujinami ${ }^{6}$ \\ ${ }^{1}$ Department of Basic Science, Graduate School of Arts and Sciences, The University of Tokyo. 3-8-1 Komaba, Meguro-ku, Tokyo 153-8902, \\ Japan. \\ ${ }^{2}$ Department of Bioinformatics, Graduate School of Medicine, Chiba University. 1-8-1 Inohana, Chuo-ku, Chiba 260-8670, Japan. \\ ${ }^{3}$ Precursory Research of Embryonic Science and Technology (PRESTO), Japan Science and Technology Agency (JST). 4-1-8 Honcho, \\ Kawaguchi, Saitama 332-0012, Japan. \\ ${ }^{4}$ Department of Biotechnology, Faculty of Life Science and Biotechnology, Fukuyama University. 1 Sanzo, Gakuen-cho, Fukuyama, Hiroshima \\ 729-0292, Japan. \\ ${ }^{5}$ Department of Computational Intelligence and Systems Science, Interdisciplinary Graduate School of Science and Engineering, Tokyo Institute \\ of Technology. 4259-G3-53 Nagatsuta-cho, Midori-ku, Yokohama, Kanagawa 226-8502, Japan. \\ ${ }^{6}$ Department of Applied Chemistry and Biotechnology, Graduate School of Engineering, Chiba University. 1-33 Yayoi, Inage, Chiba 263-8522, \\ Japan. \\ ${ }^{7}$ Department of Mathematical and Physical Sciences, Japan Women's University. 2-8-1 Mejirodai, Bunkyo-ku, Tokyo, 112-8681, Japan. \\ ${ }^{8}$ Department of Applied Chemistry, Faculty of Science and Technology, Keio University. 3-14-1 Hiyoshi, Kohoku-ku, Yokohama, Kanagawa 223- \\ 8522, Japan.
}

\begin{abstract}
This review briefly summarizes recent developments in the construction of biologically/ environmentally compatible chemical machinery composed of soft matter. Since environmental and living systems are open systems, chemical machinery must continuously fulfill its functions not only through the influx and generation of molecules but also via the degradation and dissipation of molecules. If the degradation or dissipation of soft matter molecular building blocks and biomaterial molecules/polymers can be achieved, soft matter particles composed of them can be used to realize chemical machinery such as selfpropelled droplets, drug delivery carriers, tissue regeneration scaffolds, protocell models, cell-/tissuemarkers, and molecular computing systems.
\end{abstract}

Key words: interfacial tension, quasi-elastic laser scattering method, environmentally benign surfactant, biocompatible polymer, emulsion, micelle, vesicle, protocell model, drug delivery carrier, cell/tissue maker, DNA computing, dissipation system

\section{Introduction}

Micelles, emulsion droplets, vesicles, liquid crystals, polymer fibrils, and gels are characterized as soft matter and have drawn significant attention due to their ability to form integrated molecular systems. Such self-assembled molecules and/or polymer networks interact with each other via non-covalent interactions and thermally fluctuate more than those in the solid state. Soft matter can thus adaptively respond to environmental stimuli or perturba- tions ${ }^{1)}$. When self-assembled molecules and/or polymer networks form heterogeneous interfaces or membranes, they exhibit additional long-range interactions, and environmental stimuli and perturbations may have an effect in the range from nanometers to millimeters ${ }^{2}$. Therefore, non-covalent and long-range interactions of molecular building blocks shed light on the architecture of highly organized chemical machinery with functions including multimodal responses to stimuli, autonomous motion, and

*Correspondence to: Taro Toyota, Department of Basic Science, Graduate School of Arts and Sciences, The University of Tokyo, 3-8-1 Komaba, Meguro-ku, Tokyo 153-8902, Japan.

E-mail: cttoyota@mail.ecc.u-tokyo.ac.jp

Accepted September 22, 2014 (received for review August 25, 2014)

Journal of Oleo Science ISSN 1345-8957 print / ISSN 1347-3352 online

http://www.jstage.jst.go.jp/browse/jos/ http://mc.manusriptcentral.com/jjocs 


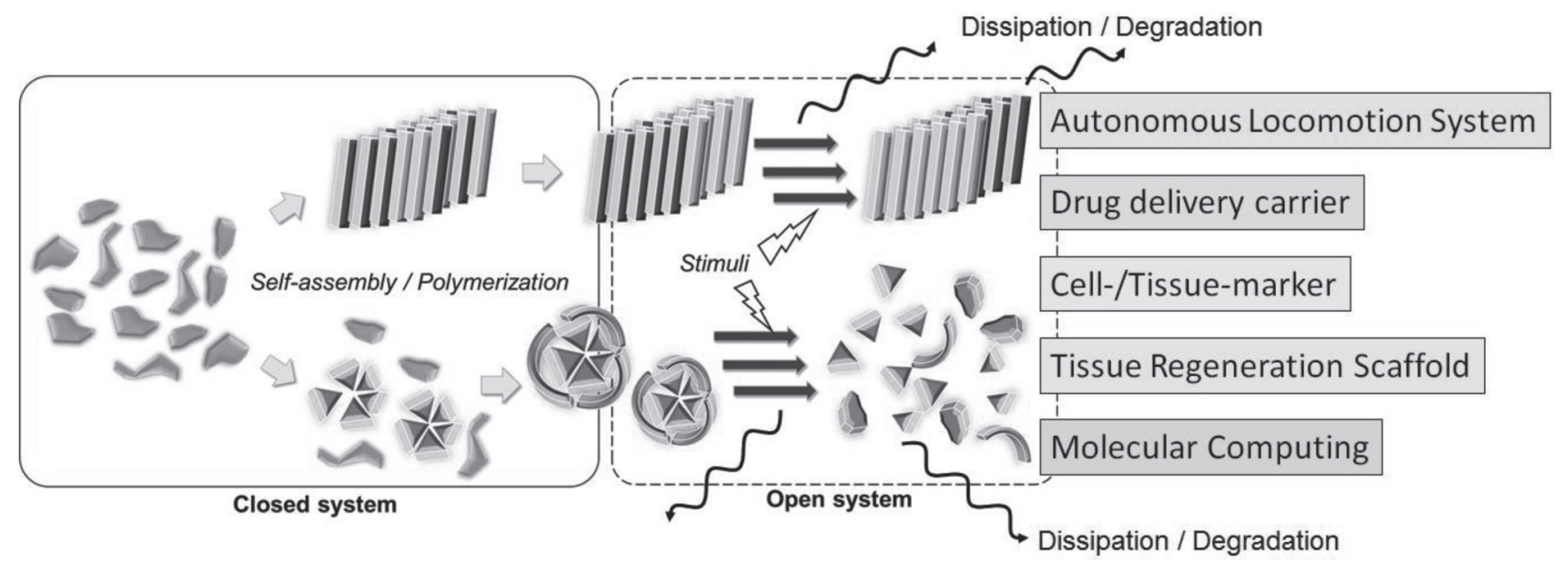

Fig. 1 Schematic illustration of chemical machinery made of soft matter working in an open system.

chemical computing with reaction circuits.

The molecular design of soft matter building blocks is conventionally conceived based on the self-assembly of molecules and/or polymers in a closed, equilibrium state. However, such highly organized chemical machinery must functionalize its output according to the environmental input in an open, energy dissipative system(Fig. 1). For example, drug delivery carriers maintain their drug-encapsulating boundaries until they have accumulated at target tumors or tissues, at which point they release the drugs, and finally the carrier building blocks are decomposed via biological reactions. Biomolecules or biologically/environmentally degradable synthetic molecules have been developed for pre-practical/practical applications such as pharmaceutics or green and sustainable chemistry. They exhibit a high holding stability until they are exposed to specific stimuli, such as enzymes, in an open system. DNA/RNA self-assembly and the corresponding reaction networks, including degradation pathways, have been utilized in the regulation of such functions. Moreover, such dissipative systems occasionally exhibit spatiotemporal pattern formation, which induces the locomotion of soft matter particles. Even though our understanding of such dynamic systems has progressed through the development of mathematical and physical models, analytical methodologies are needed to fully understand the parameters and are critical to the progress of design of soft matter building blocks and their architecture. Here, we review recent progress regarding the dynamics of biocompatible or environmentally benign soft matter and its pre-practical/practical applications by describing novel measurement techniques and soft matter building blocks.

\section{Interfacial tension: a long range interaction that sustains soft matter boundaries and potentially drives locomotion}

In an open system, the boundaries of soft matter particles are defined by their interfaces. To evaluate how the interfaces of soft matter particles sustaining their body structure and for construction of chemical machinery, it is important to understand the parameters of the structures and reactions at the molecular scale, their motions at the fluid-dynamics scale, and their long-range interactions via electrical potentials ${ }^{2)}$. For example, the motion of a selfpropelled camphor particle or alcohol droplet on an aqueous surface is known as autonomous chemical locomotion. Mathematical and physical models related to alteration of surface tension via the dissipation of camphor or alcohol molecules have been proposed, however, reports on the in situ measurement of the parameters of the dynamic system remain insufficient, and a complete understanding of these aspects of soft matter particles has yet to be established. Thus, analytical methodologies are critical to the progress of such research.

Since the number of molecules at the interface is much smaller than the number of bulk molecules, interfacial signals are easily masked by bulk signals, and thus interface-sensitive analytical methods are important in evaluating layers of molecules at interfaces. For example, atomic force microscopy (AFM) is an established method for the detection of topography, force, and other molecular-scale mechanical properties, and AFM measurements of immersed liquids have been reported ${ }^{3-6)}$. Even-order non-linear spectroscopy methods, such as second-harmonic generation $(\mathrm{SHG})^{7,8)}$ and sum frequency generation $(\mathrm{SFG})^{9,10)}$, have also been applied to detect molecular structures or orientations at interfaces, because even-order non-linear polarizations are only induced in anisotropic regions such as interfaces. Additionally, the orientation of hyperpolarization is fixed according to the molecular coordinates. SFG is 
typically used to detect molecular vibrations and the orientation of adsorbed molecules via input from infrared light ${ }^{11-13)}$. However, SFG does not afford stable signals for interfaces immersed in water due to infrared absorption, but SHG can be used with immersed interfaces in water. Hence, SHG microscopy ${ }^{14)}$ has been applied to monitoring of anisotropic phenomena such as membrane potential ${ }^{15-17)}$. In order to obtain interface information in regions smaller than the diffraction limit, a combination of optical methods and AFM, known as near-field scanning optical microscopy (NSOM, or $\mathrm{SNOM}$ ), has been developed ${ }^{18-20)}$. Using near-field illumination around a probe tip, optical absorption, fluorescence, vibrational resonances (such as tip-enhanced Raman scattering), or other electronic resonances can be detected. Because the probe area is limited to the area near the tip apex when using these methods, surface-sensitive methods using NSOM (or SNOM) have also been reported ${ }^{21-23)}$.

Although numerous methods have been established to determine the molecule-scale properties of interfaces, analytical methods that yield information between the molecular and fluid-dynamical scales are rather limited. Interfacial tension is a parameter that is affected by molecular behaviors at interfaces, and it directly drives the fluid-dynamic motions at interfaces. Interfacial tension is conventionally measured using a probe (e.g., the Wilhelmy plate method and the Du Noüy ring method) or by deforming interfaces (e.g., the pendant-drop method and the capillary-rise method). However, non-invasive and non-perturbing methods are inevitably important for the characterization of phenomena that accompany the dynamics of soft-matter interfaces, especially convectional motions. Among the approaches available to measure interfacial tension, the frequency of capillary waves at an air/liquid or liquid/liquid interface can be measured using quasi-elastic laser scattering (QELS) method ${ }^{24-27)}$ or laser induced surface deformation spectroscopy ${ }^{28)}$. QELS method is based on the angle- and frequency-resolved detection of light scattering by capillary waves, and enables the detection of interfacial tension by irradiation with laser light without contact probes. By applying this method to time- and position-resolved measurements, QELS method becomes a powerful tool in the analysis of the driving forces behind the mechanical motions derived from the instability of interfacial tension. For example, the oscillatory flip motions of oil/water interfaces in the presence of surfactants, the surface wave of Belousov-Zhabotinsky reaction solutions, and self-propelled objects at the air/water interface have all been clarified in regard to the time course of interfacial tension ${ }^{29-34)}$. The surface tension distributions around a self-propelled camphor boat and an alcohol droplet with a constant velocity are shown in Fig. 2. Whereas the camphor boat, which is a self-propelled floating plate with solid camphor tablets attached at its edges, is driven by the surface tension differences between its front and rear edges, the alcohol
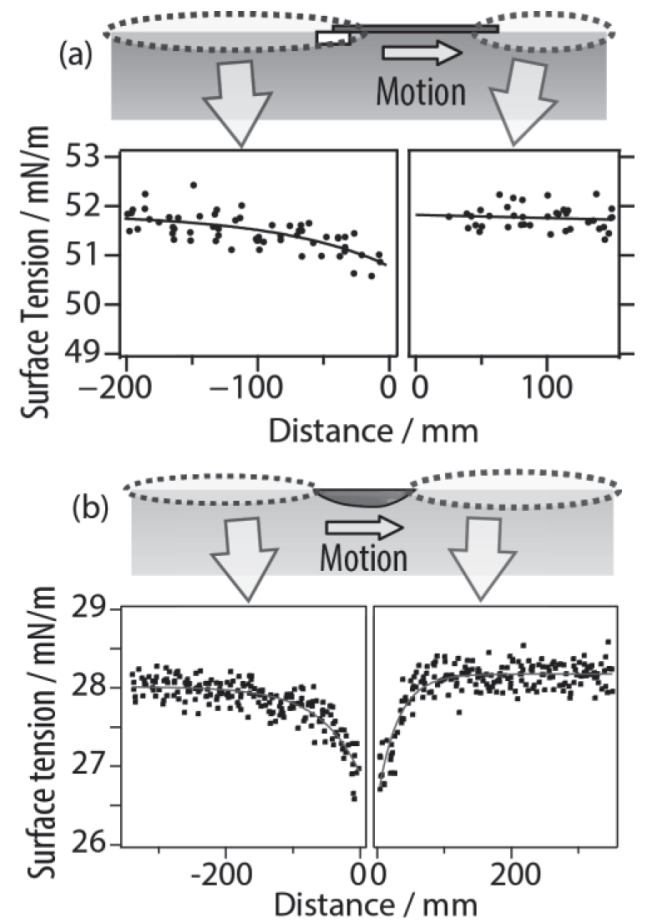

Fig. 2 (a) Spatial surface tension distributions at the rear and front of a floating camphor boat with a constant velocity of $6.5 \mathrm{~mm} / \mathrm{s}$. (b) Spatial surface-tension distributions at the rear and front of a floating alcohol droplet with a constant velocity of $15 \mathrm{~mm} / \mathrm{s}$. The origin of the horizontal axis indicates the edge position of the camphor boat or the alcohol droplet. Positive and negative distances are associated with the front and rear, respectively ${ }^{27)}$.

droplet is self-propelled by the surface tension gradient associated with the so-called Marangoni effect. The Marangoni effect is a long-range interaction between surface-active molecules and solvent molecules and is evoked at interfaces with heterogeneous interfacial tension to afford tangential flow to yield a homogeneous interfacial tension ${ }^{35-37)}$. Because the alcohol droplet has an anisotropic shape, differences in the surface tension gradient occur around it. Hence, the stronger tangential flow at the front edge causes the alcohol droplet to move. Since the alcohol droplet is consumed during its self-propelled motion and the dissolved alcohol in the bulk water is evaporated under the ambient air pressure, the system represents an open system that exhibits self-propelled motion until the alcohol droplet breaks through its boundaries. 


\section{Environmentally benign surfactants and their emulsification}

Inhomogeneous adsorption of surfactant molecules on an interface leads to Marangoni convection. Therefore, chemical machinery can also be constructed using surfactants. The emulsification of oil in aqueous systems using surfactants is well developed and has the potential to yield locomotive systems. Oil-in-water emulsion droplets have been shown to exhibit autonomous locomotion in an aqueous solution of ester-containing cationic surfactants ${ }^{38)}$. The driving force behind the autonomous locomotion was attributed to Marangoni instability around the droplets ${ }^{39)}$. Although the precise mechanism of the underwater locomotion of the oil droplets remains to be clarified, the locomotion time of the emulsion droplets was extended via chemical reactions of the surfactants ${ }^{38,40)}$.

Surfactants are not easily decomposed in the natural environment. As such, they are widely used in large quantities in industry as fibers, pharmaceutical agents, foods, and as household detergents. Owing to their solubility in water, surfactants are generally difficult to recover or reuse; therefore, if they are not biodegradable they are typically released into the environment as drainage. Therefore, the development of surfactants with improved biodegradability is required to further novel developments in green and sustainable chemistry(Fig. 3 ${ }^{41)}$. Moreover, the development of surfactants with superior performance can reduce their consumption. Figure 4 shows the molecular structures of some typical environmentally benign surfactants. Some environmentally benign surfactants have been synthesized using renewable resources, such as sugars and amino acids, and exhibit low toxicity and high biodegrad- ability. Because sugar- and amino acid-based surfactants form relatively stable emulsions with large quantities of oil components and water, they are used as emulsifiers in foods and cosmetics, in addition to their use in technical fields such as emulsion polymerization ${ }^{42,43)}$. Additionally, sugar-based biosurfactants produced by a variety of microorganisms exhibit unique properties such as mild production conditions, multifunctionality, and high environmental compatibility $^{44)}$. These features should broaden their range of applications in novel advanced technologies.

Surfactants containing hydrolyzable bonds, synthesized with fatty acids or fatty alcohols are also considered to be environmentally benign, because they exhibit excellent biodegradability by environmental microbes ${ }^{45)}$, due to the high biodegradability of their degradation compounds produced by the enzymatic hydrolysis of their linkages. Because amide-containing cationic surfactants are rapidly biodegraded by activated sludge and can solubilize oil-soluble materials in water, they have attracted much attention as hair conditioners ${ }^{46,47)}$. Biodegradable diethyl ester-type cationic surfactants have also been used as fabric soften$\mathrm{ers}^{48)}$. Furthermore, carbonate-containing surfactants are synthesized via a halogen-free green process and exhibit good biodegradability and hydrolytic stability ${ }^{49)}$.

Since the natural environment is an open system, emulsification processes using next-generation surfactants in the non-equilibrium state lie on the frontier of surfactant research, and further development of novel designs and functions of surfactants for chemical machinery is required.

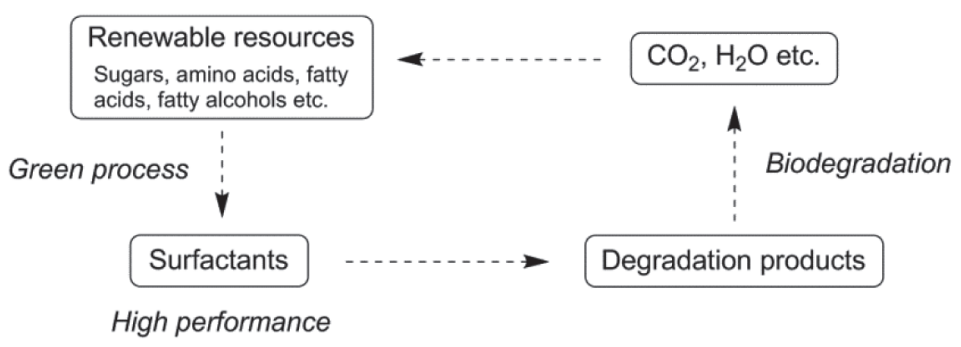

Fig. 3 Simplified conceptual scheme of next-generation, environmentally benign surfactants.

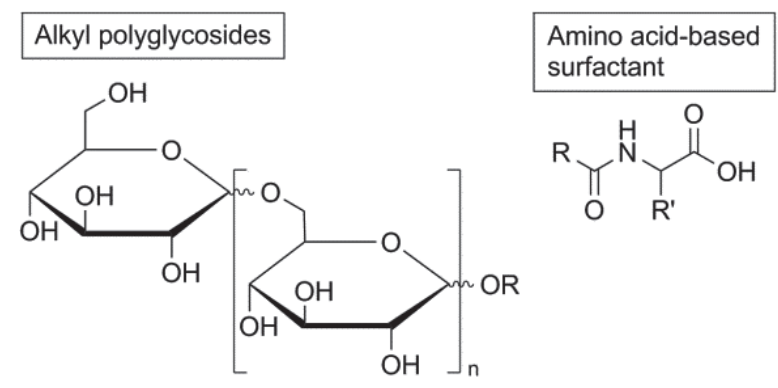

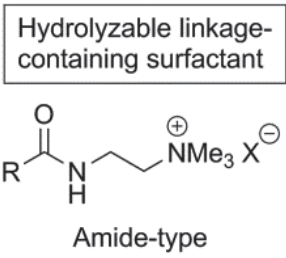

Amide-type

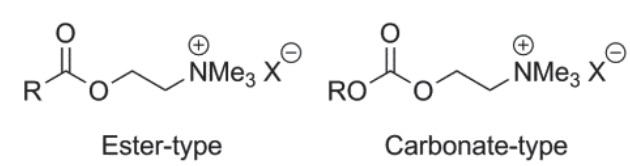

$\mathrm{R}: \mathrm{C}_{\mathrm{n}} \mathrm{H}_{2 \mathrm{n}+1}, \mathrm{X}: \mathrm{Cl}, \mathrm{Br}, \mathrm{I}$

Fig. 4 Molecular structures of typical, environmentally benign surfactants. 


\section{Biocompatible polymers for tissue regeneration scaffolds and drug-delivery vehicles}

Living bodies, including the human body, are another open system where functional chemical machinery composed of soft matter can be applied for pharmaceuticals and tissue engineering. Pharmaceutical research, especially drug delivery systems (DDSs), has drawn much attention for carrying effective drugs to tumors in humans and animals with minimal side effects. Many reviews of the DDS development have been published ${ }^{50)}$. Additionally, advanced medical treatments require advanced technologies, i.e., tissue engineering. Tissue engineering is a promising methodology for the regeneration of tissues and organs that have been subject to accidents or diseases. To regenerate tissues, the utilization of biomaterials as scaffolds or carriers of drugs, genes, or proteins is critical. Synthetic biomaterials are advantageous in that their physicochemical properties such as biocompatibility, degradability, hydrophilicity, and stiffness are easily controlled and their production is largely scalable. Aliphatic polyesters such as polylactides, polyhydroxyalkanoates and poly(e-caprolactone), polycarbonates, polyamides, polyphosphoesters, polyanhydrides, and polyorthoesters are some biodegradable synthetic polymers that have been used as biomaterials (Fig. 5) ${ }^{51)}$.

When designing new biomaterials for applications in tissue engineering, the biocompatibility of the material must be considered. In particular, the cytotoxicity of the degradation products is critical in assessing the potential of the substrate as an implantable biomaterial. Degradable polymeric biomaterials are often based on ester or amide polymer backbones, including polylactides, polyhydroxyalkanoates, and polyamines, as ester or amide hydrolysis provides a mechanism for degradation. A significant drawback of polyesters and polyamides is the formation of carboxylic acids as degradation products. As a result, the acidity of the surrounding tissue increases, and the rise in local acidity has been implicated in further scaffold degradation (self-catalyzed degradation) and pronounced inflammatory responses.

To prevent the release of acidic degradation products, a novel class of biomaterials that contain a cyclic acetal moiety as the degradable segment has been designed and synthesized. Cyclic acetals degrade under mildly acidic conditions to produce hydroxyl and carbonyl terminals as degradation products, and therefore may not affect the local acidity or promote inflammation. Recently, a series of cyclic acetal-based biomaterials for use in tissue engineering was reported ${ }^{52,53)}$. A brief description of cyclic acetalbased hydrogels for cell encapsulation and polymeric micelles for controlled drug release follows.

The encapsulation of bone marrow stromal cells (BMSCs) in hydrogels and the subsequent incubation of these hydrogels in osteogenic medium promote the proliferation and differentiation of stem cells and represent an effective therapy to regenerate bone-like tissues. Degradable hydrogels based on a polyether, poly[poly (ethylene glycol)-co-cyclic acetal] (PECA), consisting of a cyclic acetal as the degradable segment and poly (ethylene glycol) (PEG) as the hydrophilic segment were designed and produced for cell encapsulation (Fig. 6a $)^{52,54,55)}$. Encapsulation of BMSCs in PECA hydrogels was achieved via chemical crosslinking of the acryl terminal groups of PECA in the presence of BMSCs in the culture medium. The viability of BMSCs embedded on PECA hydrogels was determined using a live/dead assay; high viability was maintained for two weeks. Moreover, gene expression analysis confirmed that BMSCs embedded in PECA hydrogels exhibited osteogenic differentiation following incubation in osteogenic medium for two weeks (Fig. 6b). The degradation kinetics of the cyclic acetal segments were investigated under acidic conditions and simulated physiological conditions. The degradation rate of the cyclic acetal-based polymer under acidic conditions was significantly influ-<smiles>CCC(C)C(=O)OC</smiles>

Polylactide<smiles>[R]C(CC(C)=O)OC</smiles>

Polyhydroxyalkanoate<smiles>COC(=O)C(C)CC(C)C</smiles>

$\operatorname{Poly}(\varepsilon$-caprolactone)<smiles>CCOPOC(=O)C(C)O</smiles>

Polycarbonate<smiles>CCNNC(=O)N[R]C(=O)C(C)(C)O</smiles>

Polyamide<smiles>[R]OP(=O)(OOCC)C(C)(C)O</smiles>

Polyphosphoester<smiles>CC(C)(O)C(=O)OC(=O)C(C)(C)O</smiles>

Polyanhydride

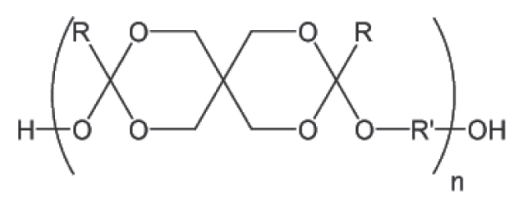

Polyorthoester

Fig. 5 A series of biodegradable synthetic polymers that have been used as biomaterials. 
(a)

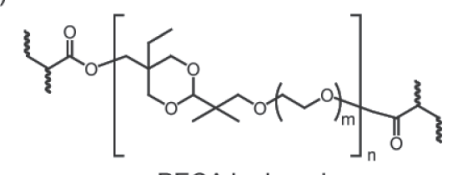

PECA hydrogel

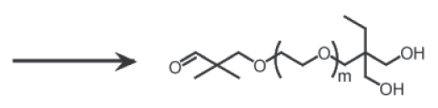

Main degradation products (b)

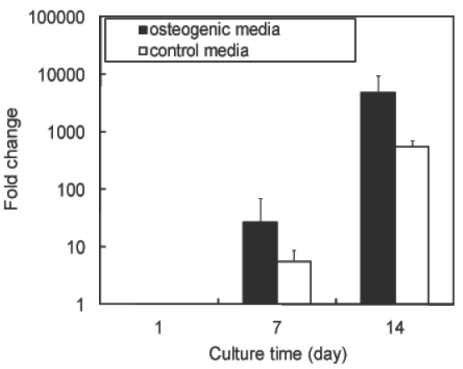

(c)

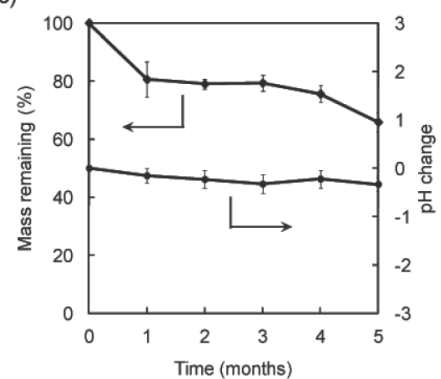

Fig. 6 (a) Poly[poly(ethylene glycol)-co-cyclic acetal] (PECA) hydrogel and its degradation product. (b) Quantitative reverse transcription-polymerase chain reaction (RT-PCR) analysis of osteocalcin expression by BMSCs encapsulated in PECA hydrogels and incubated in osteogenic or control medium for two weeks. (c) Mass remaining and $\mathrm{pH}$ change during degradation of PECA hydrogels in PBS at $37^{\circ} \mathrm{C}$.

(a)

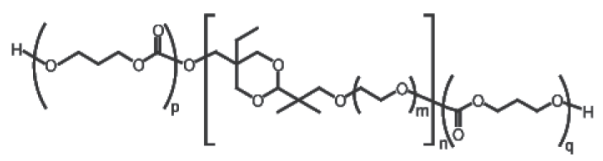

PTMC-b-PECA-b-PTMC triblock copolymer (b)

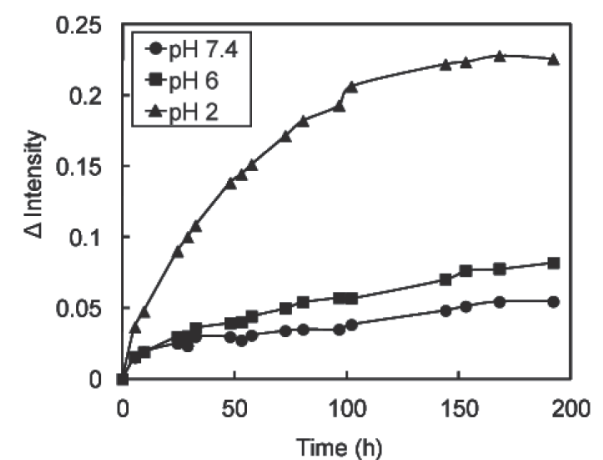

Fig. 7 (a) PTMC- $b$-PECA-b-PTMC triblock copolymer for the formation of amphiphilic polymeric micelles. (b) pHdependent controlled release of a model drug (methotrexate) from PTMC- $b$-PECA- $b$-PTMC-based polymeric micelles.

enced by $\mathrm{pH}$ and temperature. The $\mathrm{pH}$ of the buffer remained constant, and the hydrogel lost approximately $30 \%$ of its dry weight after 5 months of in vitro incubation(Fig. 6c). These results indicated that cyclic acetal-based hydrogels may be utilized as scaffolds for stem cell differentiation as their degradation products have low cytotoxicity.

Polymeric micelles are often used as drug delivery carriers due to their high stability, biocompatibility, and low critical micelle concentration. The mechanism of micelle formation is based on the hydrophilic/hydrophobic interactions of amphiphilic di- or tri-block copolymers, and is known as self-assembly. Water-insoluble drugs are entrapped within the hydrophobic core and become soluble in water because of the hydrophilic shell. The drugs are then released from the inner core of the polymeric micelle at targeted sites within the body, facilitated by the stimulisensitive structure of the micelles. Tri-block copolymers composed of PECA as the hydrophilic degradable segment and poly (trimethylene carbonate) (PTMC) as the hydrophobic segment have been synthesized, and a drug was successfully entrapped in the amphiphilic polymeric micelle (Fig. 7a ${ }^{56)}$. PTMC was chosen as the hydrophobic segment because its degradation products are non-acidic. The cyclic acetal segments were degraded under acidic conditions, whereas the carbonate linkages did not degrade under non-enzymatic conditions. The degradation rate of the copolymer was enhanced as the acidity of the buffer increased, due to the higher degradability of the cyclic acetals under acidic conditions. A drug release study using a hydrophobic drug showed that the amount of release increased as the $\mathrm{pH}$ of the buffer decreased due to the degradation of the cyclic acetal segment under acidic conditions, which led to the utilization of PECA-based micelles as a pH-sensitive DDS carriers (Fig. 7b). Cyclic acetalbased biomaterials have been shown to be viable alternatives to other biomaterials that have been used in the biomedical field. In addition to hydrogels and polymeric micelles, cyclic acetal-based polymers may also be applied as adhesives, nanospheres, and organic/inorganic hybrid materials. 


\section{Giant vesicles and their applications as protocell models and bioimaging markers}

A vesicle is a compartment structure composed of a lipid bilayer membrane. Vesicles are classified according to size. Small and large vesicles have diameters of less than 100 $\mathrm{nm}$ and in the range of $10-1000 \mathrm{~nm}$, respectively. They are also utilized as drug delivery carriers ${ }^{57)}$. Giant vesicles (GV) with diameters greater than $1 \mu \mathrm{m}$ have drawn much attention as model cell-sized biomembranes due to their size and membrane structure. Lecithin is conventionally used as a GV-forming molecule. Lecithin is a mixture of phosphocholines extracted from egg yolk or soybeans and it is used as a food additive for emulsification and stabilization of emulsions. In 1969, Reeves et al. prepared GVs from lecithin (phosphatidylcholines) from egg yolk by swelling thin films in an aqueous solution ${ }^{58,59)}$. Today, several methods are used to prepare GVs; swelling phospholipid films under alternative electric fields ${ }^{60,61)}$, fusion of small vesicles $^{62,63)}$, lipid-coating of ice droplets ${ }^{64)}$, lipid-coating of water-in-oil-in-water (W/O/W) droplets by microfluidics ${ }^{65-68)}$, and water-in-oil (W/O) emulsion transfer ${ }^{69)}$. Since each method has certain advantages and disadvantages, the most suitable preparation method should be chosen. In this section, the W/O emulsion transfer method for the construction of complex biomembrane models is described. This method was primarily investigated by Pautot et al. for the formation of unilamellar GVs. It consists of two steps, as shown in Fig. 8. The first step involves layering the aqueous phase, oil phase (1st oil phase) containing phospholipids, and W/O emulsion(2nd oil phase), which is stabilized by phospholipids. The second step involves sub-

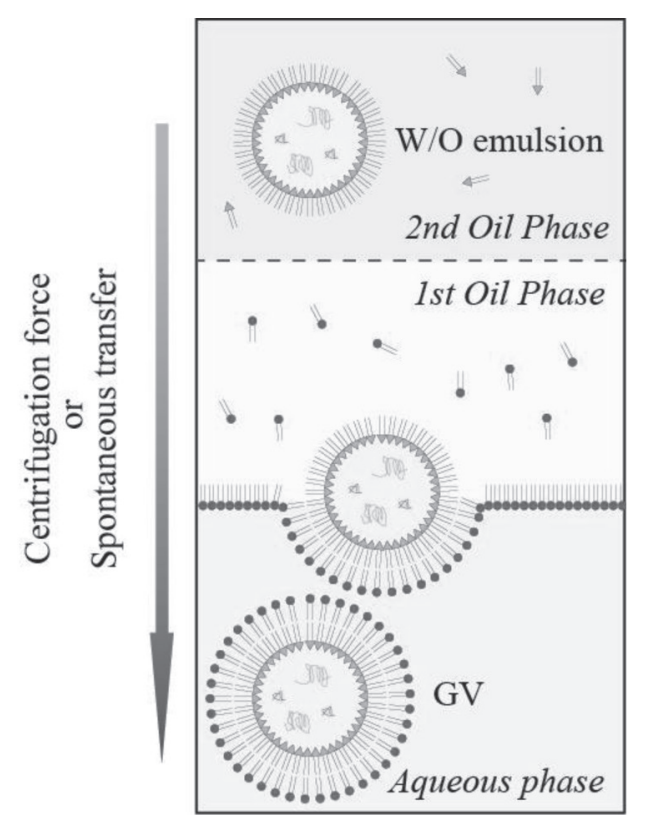

Fig. 8 Schematic illustration of the W/O emulsion transfer method. merging the $\mathrm{W} / \mathrm{O}$ emulsion droplets in the lipid monolayer on the water-oil interface by centrifugation. The outer and inner leaflets of GVs are composed of phospholipids in the 1st and 2nd oil phases. This method has several advantages, including production of GVs of the uniform lamellarity, as revealed by population analysis ${ }^{70)}$, preparation of "asymmetric" GVs with inner and outer leaflets consisting of different lipids ${ }^{71,72)}$, encapsulation of polymers and microbeads into GVs with high volume fractions ${ }^{73-75)}$, and the ability to control the size of the $\mathrm{GVs}^{76)}$.

Various studies have been conducted on protocell models constructed using the W/O emulsion transfer method. These studies have focused on gene expression and metabolic reaction networks in GVs and the control of deformations of GV membranes. As examples of the former investigations, Noireaux et al. successfully expressed green fluorescent protein ${ }^{77)}$. Nishimura et al. investigated the effect of the size and composition of GVs on protein synthesis $^{78)}$. Pontani et al. reconstituted the actin cortex ${ }^{79)}$. Fujii et al. synthesized membrane proteins ${ }^{80)}$. Hamada et al. expressed pheromone receptors ${ }^{81)}$. Moreover, cell-free translation systems ${ }^{82,83)}$ and a minimum gene expression system $^{84)}$ have been constructed in GVs. GV deformation represents the other achievement of protocell modelling. Osawa et al. demonstrated the autonomous division of GVs by the reconstitution of the $\mathrm{Z}$ ring in $\mathrm{GVS}^{85)}$. Romer et al. realized the endocytosis of GVs without clathrin ${ }^{86)}$. Terasawa et al. reported the spontaneous budding of GVs via encapsulation of a high concentration of polyethylene glycol $^{87)}$. This trend shows the acceleration of the construction of protocell models that exhibit adaptability to open systems and their applications.

Imaging of cell- or tissue-markers based on GVs serves as an example of the potential applications. The development of new technologies for imaging of living bodies or organs using cell- and tissue-markers to ensure good spatial resolution, sensitivity, and detectability is one of the most important subjects in pharmaceutical and medical treatment research. High-field magnetic resonance imaging (MRI) with contrast medium is already used clinically, and the design of new MRI probes for pharmaceutical and medical applications has attracted significant attention. Super-paramagnetic iron oxide (SPIO) is a promising candidates for the development of smart and functional probes. The use of large SPIO particles $(>1 \mu \mathrm{m})^{88)}$ may induce long-term toxicity, and, hence, smaller SPIO particles, especially nanometer-sized particles, is desirable for use in cell tracking. However, their use results in a loss of signal detectability, as well as in the accumulation of particles in cells, which may be toxic. These issues must be overcome before safe and low-toxicity cell-tracking applications can be realized. Hence, GVs that encapsulate SPIO particles at high number density have become a research focus ${ }^{89)}$. Phospholipid membranes are natural membranes that are 

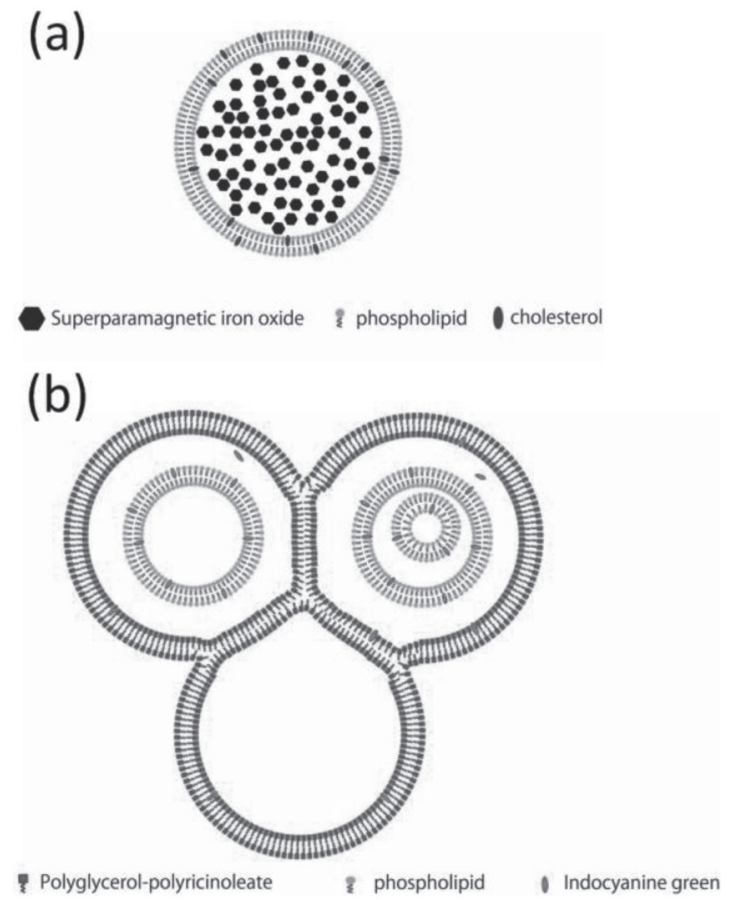

Fig. 9 Schematic illustration of (a) SPIO-GV as a cellmarker and (b) NIR-fluorescent dye as a tissuemarker.

degradable in vivo. The encapsulation of SPIO particles at a high number density in GVs yields SPIO-containing GVs (SPIO-GVs) that can be used as highly sensitive MRI probes (Fig. 9a). The W/O emulsion transfer method has been adopted and it produces SPIO-GVs composed of a single lamellar membrane with a diameter of $4.7 \pm 2.2 \mu \mathrm{m}$. In vivo micro MRIs of medaka embryos injected with SPIO-GVs have been examined. After injection of the SPIO-GV into one cell of a 4-cell stage embryo with a glass capillary and fixing with paraformaldehyde, dark spots (typical diameter, $200 \mu \mathrm{m}$ ) were observed at the blastomeres of the injected cells. This indicated that the GVbased SPIO MRI contrast agent could be effective for enhancement of the MRI signal at the cellular level. This finding will contribute to the development of imaging techniques for use in fundamental biology studies and pre-clinical pharmaceutics.

Rapid in situ imaging of human tissues is required for surgical applications. Specifically, various techniques using preoperative or intraoperative gastrointestinal endoscopies to assist with the intraoperative localization of tumors have been proposed. However, these techniques have only scarcely provided tissue markers that can be injected from within the digestive tract by an endoscopic instrument and visualized from the outside. In addition, near-infrared (NIR) fluorescent molecules, such as indocyanine green (ICG) ${ }^{90}$, have drawn much attention because the NIR light can permeate organ tissues and can be excited and detected with a fluorescence CCD camera. Furthermore, ICG has been approved by the Food and Drug Administration in the United States. However, this fluorescent probe is subject to blur and bleaching. GV-based tissue markers, including ICG, are prepared using three steps ${ }^{91)}$ : (i) mixing of ICG with lecithin GVs;, (ii) suspension of the ICG-containing giant vesicles (ICG-GV) in an oil phase dissolving polyglycerol-polyricinoleate (PGPR), and (iii) centrifugation of the suspension layered on a buffered solution to obtain a giant polymer vesicle (polymerasome) containing ICG-GV (Fig. $9 b)$. When the tissue marker was injected into the inner gastric surface of an anesthetized pig using an endoscopic syringe and the injection site was observed using a laparoscopic fluorescence camera, the diameter of the spot blur was approximately $2 \mathrm{~cm}$ over a 5 -h period, which can be used to determine the location of early-stage tumors of several centimeters in diameter. This result demonstrated the utility of tissue markers for tumor marking, and suggests their potential to assist in navigation during surgical procedures.

\section{Chemical machinery based on DNA/RNA self- assembly and biomolecular reaction networks}

In addition to the aforementioned surfactants, lipids, and biodegradable synthetic polymers, DNA and RNA are also degradable polymeric biomaterials present in environmental and living systems. In isolation from open systems, the self-assembly and dynamics of these molecules have been developed using the hybridization and dissociation of specific sequences of single-stranded DNA/RNA and enzymes. In this section, the degradation and dissipation of such molecules are described, and a novel micrometer-sized chemical open reactor for dynamic biomolecular reaction networks is introduced.

In the last decade, DNA/RNA molecules have been used as programmable soft materials, and have contributed to the construction of elaborate nano/micrometer-sized selfassembled systems such as 2D/3D nanostructures ${ }^{92-94)}$, molecular robots ${ }^{95,96)}$, molecular computing devices ${ }^{97-100)}$,' etc. Programmability of DNA/RNA can be achieved by base sequence design, and the stability of internal and hybridized structures of DNA/RNA molecules can be predicted by thermodynamic calculation of base pairing (hydrogen bonding). The construction of self-assembled DNA/RNA systems should be based on their sequence-derived programmability, and on the "soft" properties of DNA/RNA, i.e., their hydrogen bonding-based structure formation and degradability of DNA/RNA. Since the hydrogen bonds are not very strong, the formation and deformation of base pairing can easily be controlled with environmental stimuli such as temperature, $\mathrm{pH}$, ion strength, small molecules, etc. In addition, DNA/RNA can easily be degraded with nu- 

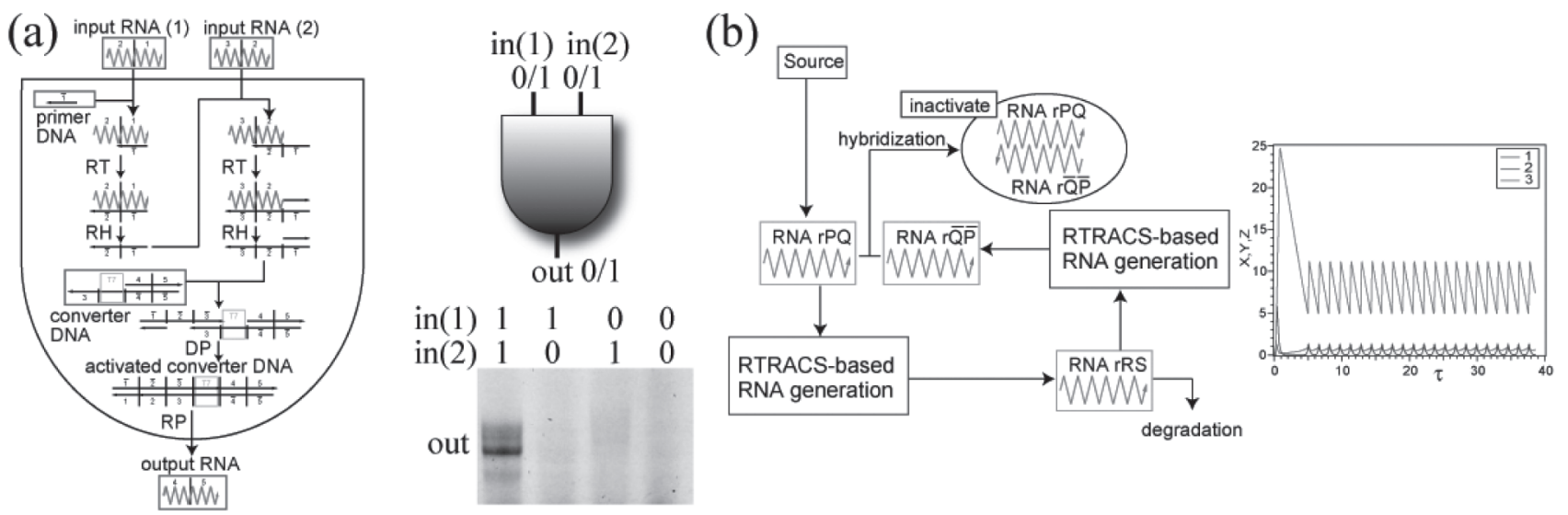

Fig. 10 (a) Schematic illustration of AND operation by RTRACS reaction and the electrophoretic observation of output RNA molecules. Output RNA was only observed when two RNAs were input. (b) Negative feedback network based on RTRACS and numerical simulations of the oscillatory reaction.
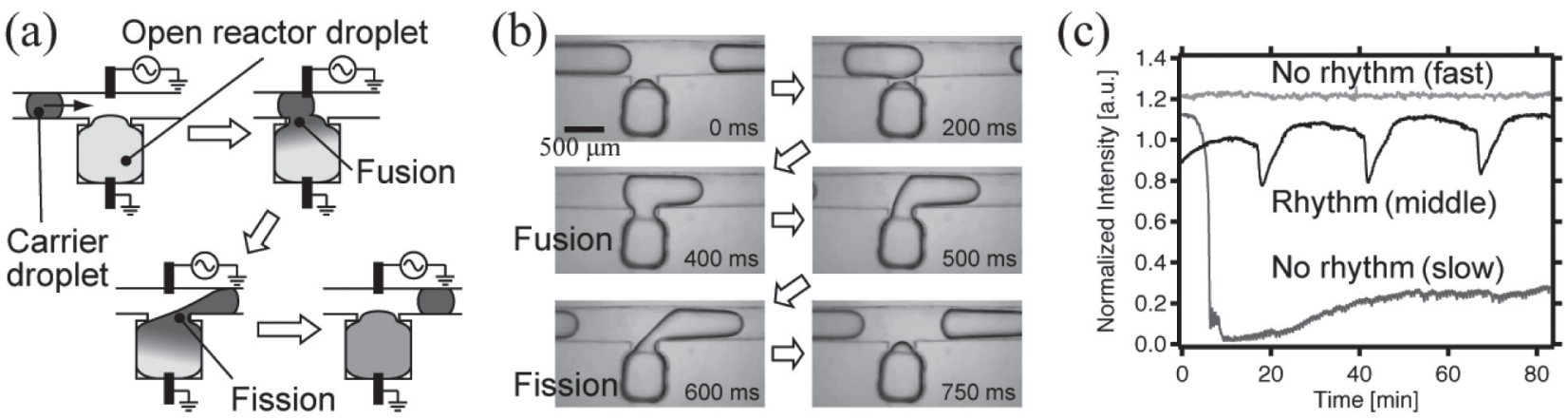

Fig. 11 (a) Conceptual illustration of microdroplet-based chemical open reactor. (b) Microscope images of the fusion and fission of droplets. (c) Sustained $\mathrm{pH}$ oscillation in the open reactor. High intensity indicates high pH. Edblom $\mathrm{pH}$ oscillation, which exhibits oscillations between $\mathrm{pH}$ approximately 7 and 3, was used.

cleases and restriction enzymes, as they are generated by polymerases. These properties of generation/formation and degradation/deformation enable time-variable dynamic functions such as robotic motion, computation, etc.

Figure 10a shows an example of a dynamic chemical system based on the generation and degradation of DNA/ RNA, called the Reverse-transcription and Transcriptionbased Autonomous Computing System(RTRACS) ${ }^{97)}$. This system exhibits logical operation, e.g., AND operation with two RNA inputs and an RNA output. When RNA molecules are input, a reverse transcriptase produces DNA; after many steps of generation, degradation, hybridization, and dissociation, an RNA polymerase finally produces an output RNA. In addition, based on this system, a molecular oscillator with periodic changes in RNA concentration can be realized (Fig. 10b ${ }^{98)}$. For the generation of oscillation, a negative feedback reaction that includes generation and degradation of RNA is essential. Similar DNA/RNA-based systems have been reported by several research groups ${ }^{99,100)}$.

In living systems, the influx and dissipation of molecules into/out of the open system are used to generate self-organized chemical dynamics, as well as to generate and degrade molecules. Self-organized dynamics are known as dissipative structures in non-equilibrium states, as proposed by Prigogine et al. ${ }^{101}$. Simultaneous with dissipation of molecules from the system, entropy is removed and order is maintained in the system. To realize a non-equilibrium state in chemical reaction systems, a microdropletbased chemical open reactor has been developed (Fig. 11a, b ${ }^{102)}$. In this open reactor, droplet fusion and fission led to the sustained influx and dissipation of molecules. Using this system, sustained rhythmic chemical oscillation ${ }^{103)}$ was achieved (Fig. 11c).

\section{Concluding remarks}

Here we have reviewed recent progress in functional soft matter particles in open systems. Surface-active agents exhibit formation of bounded liquid and emulsion droplets that are capable of self-propelled motion under certain conditions due to heterogeneous interfacial tension associated with the Marangoni effect. Examples of typical open systems include the environment and living systems. Bio- 
logically and/or environmentally compatible amphiphiles and polymers have been developed as drug delivery carriers, cell-/tissue-markers, and tissue regeneration scaffolds. Soft matter maintains its boundaries until it breaks out following appropriate adequate external stimuli. Moreover, when using information molecules such as DNA and RNA, the continuous dissipation/degradation of molecules leads to sustained dynamic behaviors in these self-assembled molecular systems such as molecular computation, rhythmic chemical reactions, etc. To construct highly functional chemical machinery composed of self-assembled molecular/polymer systems such as molecular robots ${ }^{104)}$, the dissipation or degradation of molecules as well as the influx or generation of molecules should be considered during system design.

\section{Acknowledgement}

The authors are grateful to Prof. Masami Hagiya(The University of Tokyo) for careful reading and critical reviewing the manuscript. This work was partly supported by a Grant-in-Aid for Scientific Research on Innovative Areas "Molecular Robotics" (T.T., M.T., No. 24104001-5) and the 21st Century COE Program "KEIO LCC" (S.N., S.M.) of The Ministry of Education, Culture, Sports, Science, and Technology, Japan. S.N. acknowledges a Grant-in-Aid for General Scientific Research, JSPS Fellows (20-4190). T.T. appreciates the aid from Urakami food and food Culture foundation, Hayao Nakayama Foundation for Science, Technology and Culture, and Kayamori Foundation of Informational Science Advancement.

\section{References}

1) Hamley, I. W. Introduction to Soft Matter: Polymers, Colloids, Amphiphiles, and Liquid Crystals, Wiley, Chichester (2000).

2) Doi, M. Soft Matter Physics, Oxford University Press, (2013).

3) McConney, M. E.; Singamaneni, S.; Tsukruk, V. V. Probing Soft Matter with the Atomic Force Microscopies: Imaging and Force Spectroscopy. Polym. Rev. 50, 235-286 (2010).

4) Barth, C.; Foster, A. S.; Henry, C. R.; Shluger, A. L. Recent Trends in Surface Characterization and Chemistry with High-Resolution Scanning Force Methods. Adv. Mater. 23, 477-501(2011).

5) Ando, T.; Kodera, N.; Takai, E.; Maruyama, D.; Saito, K.; Toda, A. A High-Speed Atomic Force Microscope for Studying Biological Macromolecules. Proc. Natl. Acad. Sci. U.S.A. 98, 12468-12472 (2001).

6) Fukuma, T.; Higgins, M. J.; Jarvis, S. P. Direct Imaging of Individual Intrinsic Hydration Layers on Lipid Bilayers at Ångstrom Resolution. Biophys. J. 92, 36033609 (2007).

7) Terhune, R. W.; Maker, P. D.; Savage, C. M. Optical Harmonic Generation in Calcite. Phys. Rev. Lett. 8, 404-406 (1962).

8) Shen, Y. R. The Principles of Nonlinear Optics. J. Wiley, New York (1984).

9) Zhu, X. D.; Suhr, H.; Shen, Y. R. Surface Vibrational Spectroscopy by Infrared-Visible Sum Frequency Generation. Phys. Rev. B 35, 3047-3050 (1987).

10) Arnolds, H.; Bonn, M. Ultrafast Surface Vibrational Dynamics. Surf. Sci. Rep. 65, 45-66 (2010).

11) Kett, P. J. N.; Casford, M. T. L.; Davies, P. B. Sum Frequency Generation (SFG) Vibrational Spectroscopy of Planar Phosphatidylethanolamine Hybrid Bilayer Membranes under Water. Langmuir 26, 9710-9719 (2010).

12) Ding, B.; Soblosky, L.; Nguyen, K.; Geng, J.; Yu, X.; Ramamoorthy, A.; Chen, Z. Physiologically-Relevant Modes of Membrane Interactions by the Human Antimicrobial Peptide, LL-37, Revealed by SFG Experiments. Sci. Rep. 3, 1-8(2013).

13) Liu, Y.; Jasensky, J.; Chen, Z. Molecular Interactions of Proteins and Peptides at Interfaces Studied by Sum Frequency Generation Vibrational Spectroscopy. Langmuir 28, 2113-2121 (2012).

14) Reeve, J. E.; Anderson, H. L.; Clays, K. Dyes for Biological Second Harmonic Generation Imaging. Phys. Chem. Chem. Phys. 12, 13484-13498(2010).

15) Peleg, G.; Lewis, A.; Linial, M.; Loew, L. M. Nonlinear Optical Measurement of Membrane Potential Around Single Molecules at Selected Cellular Sites. Proc. Natl. Acad. Sci. U.S.A. 96, 6700-6704(1999).

16) Nuriya, M.; Jiang, J.; Nemet, B.; Eisenthal, K. B.; Yuste, R. Imaging Membrane Potential in Dendritic Spines. Proc. Natl. Acad. Sci. U.S.A. 103, 786-790 (2006).

17) Dombeck, D. A.; Sacconi, L.; Blanchard-Desce, M.; Webb, W. W. Optical Recording of Fast Neuronal Membrane Potential Transients in Acute Mammalian Brain Slices by Second-Harmonic Generation Microscopy. J. Neurophysiol. 94, 3628-3636 (2005).

18) Dufrêne, Y. F.; Garcia-Parajo, M. F. Recent Progress in Cell Surface Nanoscopy: Light and Force in the Near-Field. Nano Today 7, 390-403 (2012).

19) van Zanten, T. S.; Cambi, A.; Garcia-Parajo, M. F. A Nanometer Scale Optical View on the Compartmentalization of Cell Membranes. BBA- Biomembranes 1798, 777-787 (2010).

20) Dickenson, N.; Armendariz, K.; Huckabay, H.; Livanec, P.; Dunn, R. Near-Field Scanning Optical Microscopy: A Tool for Nanometric Exploration of Biological Membranes. Anal. Bioanal. Chem. 396, 
31-43(2010).

21) Hartschuh, A. Tip-Enhanced Near-Field Optical Microscopy. Angew. Chem. Int. Ed. 47, 8178-8191 (2008).

22) Böhme, R.; Richter, M.; Cialla, D.; Rösch, P.; Deckert, V.; Popp, J. Towards a Specific Characterisation of Components on a Cell Surface-Combined TERS-Investigations of Lipids and Human Cells. J. Raman Spectrosc. 40, 1452-1457(2009).

23) Zhang, R.; Zhang, Y.; Dong, Z. C.; Jiang, S.; Zhang, C.; Chen, L. G.; Zhang, L.; Liao, Y.; Aizpurua, J.; Luo, Y.; Yang, J. L.; Hou, J. G. Chemical Mapping of a Single Molecule by Plasmon-Enhanced Raman Scattering. Nature 498, 82-86 (2013).

24) Katyl, R. H.; Ingard, U. Scattering of Light by Thermal Ripplons. Phys. Rev. Lett. 20, 248-249 (1968).

25) Sano, M.; Kawaguchi, M.; Chen, Y.-L.; Skarlupka, R. J.; Chang, T.; Zografi, G.; Yu, H. Technique of SurfaceWave Scattering and Calibration with Simple Liquids. Rev. Sci. Instrum. 57, 1158-1162 (1986).

26) Sakai, K.; Choi, P.-K.; Tanaka, H.; Takagi, K. A New Light Scattering Technique for a Wide-Band Ripplon Spectroscopy at the MHz Region. Rev. Sci. Instrum. 62, 1192-1195 (1991).

27) Nomoto, T.; Toyota, T.; Fujinami, M. Quasi-Elastic Quasi-Elastic Laser Scattering for Measuring Inhomogeneous Interfacial Tension in Non-Equilibrium Phenomena with Convective Flow. Anal. Sci. 30, 707716 (2014).

28) Sakai, K.; Mizuno, D.; Takagi, K. Measurement of Liquid Surface Properties by Laser-Induced Surface Deformation Spectroscopy. Phys. Rev. E 63, 046302 (2001).

29) Ikezoe, Y.; Ishizaki, S.; Takahashi, T.; Yui, H.; Fujinami, M.; Sawada, T. Hydrodynamically Induced Chemical Oscillation at a Water/Nitrobenzene Interface. $J$. Colloid Interface Sci. 275, 298-304(2004).

30) Toyota, T.; Kimura, T.; Miyoshi, K.; Fujinami, M. Time-Resolved Quasi-Elastic Laser Scattering Study Demonstrating Heterogeneity of Interfacial Tension at the Water/Nitrobenzene Interface after Introduction of Sodium Alkylsulfate. J. Colloid Interface Sci. 349, 632-636 (2010).

31) Toyota, T.; Uchiyama, K.; Kimura, T.; Nomoto, T.; Fujinami, M. Effects of Surfactants and Electrolytes on Chemical Oscillation at a Water/Nitrobenzene Interface Investigated by Quasi-elastic Laser Scattering Method. Anal. Sci. 29, 911-917(2013).

32) Oshima, S.; Nomoto, T.; Toyota, T.; Fujinami, M. Surface Tension Gradient around an Alcohol Droplet Moving Spontaneously on a Water Surface. Anal. Sci. 30, 441-444 (2014).

33) Nomoto, T.; Goto, K.; Uchiyama, K.; Toyota, T.; Fujinami, M. Spontaneous Oscillation Mechanism by Si- multaneous Time-resolved Measurements of Interfacial Tensions of Both the Donor/Membrane and Membrane/Acceptor Phases. Anal. Sci. 30, 463-469 (2014).

34) Karasawa, Y.; Oshima, S.; Nomoto, T.; Toyota, T.; Fujinami, M. Simultaneous Measurement of Surface Tension and its Gradient around a Moving Camphor Boat on a Water Surface. Chem. Lett. 43, 1002-1004 (2014).

35) Sterling, C. V.; Scriven, L. E. Interfacial Turbulence: Hydrodynamic Instability and Marangoni Effect. AIChE J. 5, 104-106 (1959).

36) Scriven, L. E.; Sterling, C. V. On cellular conversion driven by surface tension gradients - Effect of mean surface tension and surface viscosity. J. Fluid Mech. 19, 321-340 (1964).

37) Grzybowski, B. A. Chemistry in Motion: Reaction-Diffusion Systems for Micro- and Nanotechnology, Wiley, (2009).

38) Banno, T.; Kuroha, R.; Toyota, T. pH-Sensitive Selfpropelled Motion of Oil Droplets in the Presence of Cationic Surfactants Containing Hydrolyzable Ester Linkages. Langmuir 28, 1190-1195(2012).

39) Thutupalli, S.; Seemann, R.; Herminghaus, S. Swarming Behavior of Simple Model Squirmers. New J. Phys. 13, 073021 (2011).

40) Miura, S.; Banno, T.; Tonooka, T.; Osaki, T.; Takeuchi, S.; Toyota, T. pH-Induced Motion Control of Self-Propelled Oil Droplets Using a Hydrolyzable Gemini Cationic Surfactant. Langmuir 30, 7977-7985 (2014).

41) Anastas, P. T.; Warner, J. C. Green Chemistry: Theory and Practice. Oxford: Oxford University Press, (1998).

42) Foley, P.; Kermanshahi, A.; Beach, E. S.; Zimmerman, J. B. Derivation and Synthesis of Renewable Surfactants. Chem. Soc. Rev. 41, 1499-1518(2012).

43) von Rybinski, W.; Hill, K. Alkyl Polyglycosides-Properties and Applications of a New Class of Surfactants. Angew. Chem. Int. Ed. 37, 1328-1345 (1998).

44) Kitamoto, D.; Morita, T.; Fukuoka, T.; Konishi, M.; Imura, T. Self-Assembling Properties of Glycolipid Biosurfactants and their Potential Applications. Curr. Opin. Colloid Interface Sci. 14, 315-328 (2009).

45) Tehrani-Bagha, A. R.; Holmberg, K. Cleavable surfactant. Curr. Opin. Colloid Interface Sci. 12, 81-91 (2007).

46) Sakai, T.; Inoue, K.; Yamane, M.; Toyo, T.; Nishiyama, $\mathrm{N}$.; Kaneko, Y. The Excellent Water-Solubility of $N$-[3(dimethylamino) propyl] Docosanamide Chloride: The Most Eco-Friendly Cationic Hair Conditioning Agent. J. Oleo Sci. 57, 521-527(2008).

47) Yamane, M.; Toyo, T.; Inoue, K.; Sakai, T.; Kaneko, Y.; Nishiyama, N. Aquatic Toxicity and Biodegradability of Advanced Cationic Surfactant APA-22 Compatible 
with the Aquatic Environment. J. Oleo Sci. 57, 529538(2008).

48) Giolando, S. T.; Rapaport, R. A.; Larson, R. J.; Federle, T. W.; Stalmans, M.; Masscheleyn, P. Environmental Fate and Effects of DEEDMAC: A New Rapidly Biodegradable Cationic Surfactant for Use in Fabric Softeners. Chemosphere 30, 1067-1083 (1995).

49) Banno, T.; Toyota, T.; Matsumura, S. Biodegradation -Life of Science (Chamy, R. and Rosenkranz, F. Eds.) . Rijecka, Croatia: Intech, 115-140 (2013).

50) Ranade, V. R.; Cannon, J. B. Drug Delivery Systems, $3^{\text {rd }}$ ed., CRC Press (2011).

51) Ulery, B. D.; Nair, L. S.; Laurencin, C.T.; Biomedical Applications of Biodegradable Polymers. J. Polym. Sci. Part B Polym. Phys. 49, 832-864(2011).

52) Kaihara, S.; Matsumura, S.; Fisher, J. P. Synthesis and Characterization of Cyclic Acetal Based Degradable Hydrogels. Eur. J. Pharm. Biopharm. 68, 67-73 (2008).

53) Falco, E. E.; Patel, M.; Fisher, J. Recent Developments in Cyclic Acetal Biomaterials for Tissue Engineering Applications. Pharmaceut. Res. 25, 23482356 (2008).

54) Kaihara, S.; Matsumura, S.; Fisher, J. P. Synthesis and Properties of Poly[poly (ethylene glycol)-co-cyclic acetal]Based Hydrogels. Macromolecules 40, 7625$7632(2007)$.

55) Kaihara, S.; Matsumura, S.; Fisher, J. P. Cellular Responses to Degradable Cyclic Acetal Modified PEG Hydrogels. J. Biomed. Mater. Res. A 90A, 863-873 (2009).

56) Kaihara, S.; Fisher, J. P.; Matsumura, S. Chemo-Enzymatic Synthesis of Degradable PTMC- $b$-PECA- $b$-PTMC Triblock Copolymers and their Micelle Formation for pH-Dependent Controlled Release. Macromol. Biosci. 9, 613-621 (2009).

57) Lasic, D. D.; Papahadjopoulos, D. Medical Applications of Liposomes, Elsevier (1998).

58) Reeves, J. P.; Dowben, R. M. Formation and Properties of Thin-Walled Phospholipid Vesicles. J. Cell. Physiol. 73, 49-60 (1969).

59) Needham, D.; Evans, E. Structure and Mechanical Properties of Giant Lipid (DMPC) Vesicle Bilayers from 20 Below to 10 Above the Liquid Crystal-Crystalline Phase Transition at $24^{\circ} \mathrm{C}$. Biochemistry 27, 8261-8269 (1988).

60) Angelova, M. I.; Dimitrov, D. S. Liposome Electroformation. Faraday Discuss. Chem. Soc. 81, 303-311 (1986).

61) Dimitrov, D. S.; Angelova, M. I. Lipid Swelling and Liposome Formation on Solid Surfaces in External Electric Fields. Prog. Colloid Polym. Sci. 73, 48-56 (1987).

62) Oku, N.; MacDonald, R. C. Differential Effects of Al- kali Metal Chlorides on Formation of Giant Liposomes by Freezing and Thawing and Dialysis. Biochemistry 22, 855-863 (1983).

63) Ahl, P. L.; Chen, L.; Perkins, W. R.; Minchey, S. R.; Boni, L. T.; Taraschi, T. F.; Janoff, A. S. Interdigitation-Fusion: A New Method for Producing Lipid Vesicles of High Internal Volume. Biochim. Biophys. Acta 1195, 237-244(1994).

64) Sugiura, S.; Kuroiwa, T.; Kagota, T.; Nakajima, M.; Sato, S.; Mukataka, S.; Walde, P.; Ichikawa, S. Novel Method for Obtaining Homogeneous Giant Vesicles from a Monodisperse Water-in-Oil Emulsion Prepared with a Microfluidic Device. Langmuir 24, 4581-4588 (2008).

65) Shum, H. C.; Lee, D.; Yoon, I.; Kodger, T.; Weitz, D. A. Double Emulsion Templated Monodisperse Phospholipid Vesicles. Langmuir 24, 7651-7653 (2008).

66) Funakoshi, K.; Suzuki, H.; Takeuchi, S. Formation of Giant Lipid Vesicle Like Compartments from a Planar Lipid Membrane by a Pulsed Jet Flow. J. Am. Chem. Soc. 129, 12608-12609 (2007).

67) Stachowiak, J. C.; Richmond, D. L.; Li, T. H.; Liu, A. P.; Parekh, S. H.; Fletcher, D. A. Unilamellar Vesicle Formation and Encapsulation by Microfluidic Jetting. Proc. Natl. Acad. Sci. U.S.A. 105, 4697-4702 (2008).

68) Ota, S.; Yoshizawa, S.; Takeuchi, S. Microfluidic Formation of Monodisperse, Cell-sized, and Unilamellar Vesicles. Angew. Chem. Int. Ed. 48, 6533-6537 (2009).

69) Pautot, S.; Frisken, B. J.; Weitz, D. A. Production of Unilamellar Vesicles Using an Inverted Emulsion. Langmuir 19, 2870-2879(2003).

70) Nishimura, K.; Hosoi, T.; Sunami, T.; Toyota, T.; Fujinami, M.; Oguma, K.; Matsuura, T.; Suzuki, H.; Yomo, T. Population Analysis of Structural Properties of Giant Liposomes by Flow Cytometry. Langmuir 25, 10439-10443 (2009).

71) Putot, S.; Frisken, B. J.; Weitz, D. A. Engineering Asymmetric Vesicles. Proc. Natl. Acad. Sci. U.S.A. 100, 10718-10721 (2003).

72) Hamada, T.; Miura, Y.; Komatsu, Y.; Kishimoto, Y.; Vestergaard, M.; Takagi, M. Construction of Asymmetric Cell-Sized Lipid Vesicles from Lipid-Coated Water-in-Oil Microdroplets, J. Phys. Chem. B 112, 14678-14681 (2008).

73) Whittenton, J.; Harendra, S.; Pitchumani, R.; Mohanty, K.; Vipulanandan, C.; Thevananther, S. Evaluation of Asymmetric Liposomal Nanoparticles for Encapsulation of Polynucleotides. Langmuir 24, 8533-8540 (2008).

74) Natsume, Y.; Toyota, T. Giant Vesicles Containing Microspheres with High Volume Fraction Prepared by Water-in-Oil Emulsion Centrifugation. Chem. Lett. 42, 295-297(2013). 
75) Takiguchi, K.; Yamada, A.; Negishi, M.; Tanaka-Takiguchi, Y.; Yoshikawa, K. Entrapping Desired Amounts of Actin Filaments and Molecular Motor Proteins in Giant Liposomes. Langmuir 24, 11323-11326 (2008).

76) Nishimura, K.; Suzuki, H.; Toyota, T.; Yomo, T. Size Control of Giant Unilamellar Vesicles Prepared from Inverted Emulsion Droplets. J. Colloid Interface Sci. 376, 119-125 (2012).

77) Noireaux, V.; Libchaber, A. A Vesicle Bioreactor as a Step toward an Artificial Cell Assembly. Proc. Natl. Acad. Sci. U.S.A. 101, 17669-17674(2004).

78) Nishimura, K.; Matsuura, T.; Nishimura, K.; Sunami, T.; Suzuki, H.; Yomo, T. Cell-Free Protein Synthesis inside Giant Unilamellar Vesicles Analyzed by Flow Cytometry. Langmuir 28, 8426-8432 (2012) .

79) Pontani, L. L.; van der Gucht, J.; Salbreux, G.; Heuvingh, J.; Joanny, J. F.; Sykes, C. Reconstitution of an Actin Cortex Inside a Liposome. Biophys. J. 96, 192198(2009).

80) Fujii, S.; Matsuura, T.; Sunami, T.; Kazuta, Y.; Yomo, T. In Vitro Evolution of $\alpha$-Hemolysin using a Liposome Display. Proc. Natl. Acad. Sci. U.S.A. 110, 1679616801 (2013).

81) Hamada, S.; Tabuchi, M.; Toyota, T.; Sakurai, T.; Hosoi, T.; Nomoto, T.; Nakatani, K.; Fujinami, M.; Kanzaki, R. Giant Vesicles Functionally Expressing Membrane Receptors for an Insect Pheromone. Chem. Commun. 50, 2958-2961 (2014).

82) Saito, H.; Yamada, A.; Ohmori, R.; Kato, Y.; Yamanaka, T.; Yoshikawa, K.; Inoue, T. Towards Constructing Synthetic Cells: RNA/RNP Evolution and Cell-Free Translational Systems in Giant Liposomes, in Proceedings of the International Symposium on MicroNanomechanotronics and Human Science. 286-291 (2007).

83) Yamaji, K.; Kanai, T.; Nomura, S. M.; Akiyoshi, K.; Negishi, M.; Chen, Y.; Atomi, H.; Yoshikawa, K.; Imanaka, T. Protein Synthesis in Giant Liposomes using the In Vitro Translation System of Thermococcus Kodakaraensis. IEEE Trans. Nanobioscience 8, 325331 (2009).

84) Saito, H.; Kato, Y.; Berre, M. L.; Yamada, Y.; Inoue, T.; Yosikawa, K.; Baigl, D. Time-Resolved Tracking of a Minimum Gene Expression System Reconstituted in Giant Liposomes. ChemBioChem 10, 1640-1643 (2009).

85) Osawa, M.; Erickson, H. P. Liposome Division by a Simple Bacterial Division Machinery. Proc. Natl. Acad. Sci. U.S.A. 110, 11000-11004(2013).

86) Romer, W.; Pontani, L. L.; Sorre, B.; Rentero, C.; Berland, L.; Chambon, V.; Lamaze, C.; Bassereau, P.; Sykes, C.; Gaus, K.; Johannes, L. Actin Dynamics Drive Membrane Reorganization and Scission in
Clathrin-Independent Endocytosis. Cell 140, 540-553 (2010).

87) Terasawa, H.; Nishimura, K.; Suzuki, H.; Matsuura, T.; Yomo, T. Coupling of the Fusion and Budding of Giant Phospholipid Vesicles Containing Macromolecules. Proc. Natl. Acad. Sci. U.S.A. 109, 5942-5947 (2012).

88) Shapiro, E. M.; Skrtic, S.; Sharer, K.; Hill, J. M.; Dunbar, C. E.; Koretsky, A. P. MRI Detection of Single Particles for Cellular Imaging. Proc. Natl. Acad. Sci. U.S.A. 101, 10901-10906 (2004).

89) Toyota, T.; Ohguri, N.; Maruyama, K.; Fujinami, M.; Saga, T.; Aoki, I. Giant Vesicles Containing Superparamagnetic Iron Oxide as Biodegradable CellTracking MRI Probes. Anal. Chem. 84, 3952-3957 (2012).

90) Landsman, M. L.; Kwant, G.; Mook, G. A.; Zijlstra, W. G. Light-absorbing properties, stability, and spectral stabilization of indocyanine green. J. Appl. Physiol. 40, 575-583 (1976).

91) Hatayama, H.; Toyota, T.; Hayashi, H.; Nomoto, T.; Fujinami, M. Application of a Novel Near InfraredFluorescence Giant Vesicle-and Polymerasome-based Tissue Marker for Endoscopic and Laparoscopic Navigation. Anal. Sci. 230, 225-230 (2014).

92) Seeman, N. C. From Genes to Machines: DNA Nanomechanical Devices. Trends Biochem. Sci. 30, 119125 (2005).

93) Ohno, H.; Kobayashi, T.; Kabata, R.; Endo, K.; Iwasa, T.; Yoshimura, S. H.; Takeyasu, K.; Inoue, T.; Saito, H. Synthetic RNA-Protein Complex Shaped Like an Equilateral Triangle. Nature Nanotech. 6, 116-120 (2011).

94) Langecker, M.; Arnaut, V.; Martin, T. G.; List, J.; Renner, S.; Mayer, M.; Dietz, H.; Simmel, F. C. Synthetic Lipid Membrane Channels Formed by Designed DNA Nanostructures. Science 338, 932-936 (2012).

95) Murata, S.; Konagaya, A.; Kobayashi, S.; Saito, H.; Hagiya, M. Molecular Robotics: A New Paradigm for Artifacts. New Generation Computing 31, 27-45 (2013).

96) Douglas, S. M.; Bachelet, I.; Church, G. M. A LogicGated Nanorobot for Targeted Transport of Molecular Payloads. Science 335, 831-834 (2012).

97) Takinoue, M.; Kiga, D.; Shohda, K.-I.; Suyama, A. Experiments and Simulation Models of a Basic Computation Element of an Autonomous Molecular Computing System. Phys. Rev. E 78, 041921 (2008).

98) Takinoue, M.; Kiga, D.; Shohda, K.-I.; Suyama, A. RNA Oscillator: Limit Cycle Oscillations based on Artificial Biomolecular Reactions. New Generat. Comput. 27, 107-127 (2009).

99) Montagne, K.; Plasson, R.; Sakai, Y.; Fujii, T.; Ron- 
delez, Y. Programming an In Vitro DNA Oscillator using a Molecular Networking Strategy. Mol. Sys. Biol. 7, 466 (2011).

100) Kim, J.; Winfree, E. Synthetic In Vitro Transcriptional Oscillators. Mol. Sys. Biol. 7, 465(2011).

101) Nicolis, G.; Prigogine, I. Self-Organization in Nonequilibrium Systems: From Dissipative Structures to Order through Fluctuations. John Wiley \& Sons, New York (1977).

102) Takinoue, M.; Onoe, H.; Takeuchi, S. Fusion and Fission Control of Picoliter-Sized Microdroplets for
Changing the Solution Concentration of Microreactors. Small 6, 2374-2377 (2010).

103) Edblom, E. C.; Luo, Y.; Orban, M.; Kustin, K.; Epstein, I.R. Systematic Design of Chemical Oscillators. 45. Kinetics and Mechanism of the Oscillatory BromateSulfite-Ferrocyanide Reaction. J. Phys. Chem. 93, 2722-2727 (1989).

104) Hagiya, M.; Konagaya, A.; Kobayashi, S.; Saito, H.; Murata, S. Molecular Robots with Sensors and Intelligence. Acc. Chem. Res. 47, 1681-1690(2014). 